Article

\title{
The needs of traumatic brain injury survivors' caregivers and the implication required during the COVID-19 pandemic: Public health issues
}

\author{
Hamidah Othman, ${ }^{1}$ Salizar Mohamed Ludin, ${ }^{1}$ Sanisah Saidi, ${ }^{2}$ Mohamed Saufi Awang ${ }^{3}$ \\ ${ }^{1}$ Critical Care Nursing Department, Kulliyyah of Nursing, International Islamic University Malaysia (IIUM), \\ Kuantan, Pahang; ${ }^{2}$ Medical-Surgical Nursing Department, Kulliyyah of Nursing, International Islamic \\ University Malaysia (IIUM), Kuantan, Pahang; ${ }^{3}$ Kulliyyah of Medicine, International Islamic University \\ Malaysia (IIUM), Kuantan, Pahang, Malaysia
}

\begin{abstract}
Background: Traumatic brain injury (TBI) survivors require attention and dependence from their primary caregiver. This is because TBI is a defect that affects both the psychological and physical functions of the victim. Caregivers play an essential role in providing the adequate care victims need to adjust to the new problems they may experience due to their condition, as family members alone may not be able to provide for their needs. In particular, TBI caregivers may face specific challenges when assisting their patients in handling unexpected changes to their daily routines. Therefore, this quantitative study aims to explore the needs of caregivers, their coping mechanisms towards stressful and traumatic circumstances, and how they provide care to their loved ones during the COVID-19 pandemic. Design and Methods: It was conducted with ten caregivers of individuals suffering from traumatic brain injury, that were selected using a theoretical sampling method. Data were obtained using a semi-structured interview guide, which helped the caregivers provide their responses. Meanwhile, data analysis was performed using the NVIVO analysis software. Results: The results showed that there were, three significant themes namely, (a) Support needed, (b) the information need for care, and (c) developing self-resilience. The results also showed that caregivers really need support from the various parties, and the participants lack information on specific care techniques for the severe traumatic brain injury (TBI) survivors. Conclusions: In conclusion, caregivers require approval and seek more useful information to provide excellent care to their loved ones. Being aware of the caregiver's needs would enable them to offer improved customized care.
\end{abstract}

\section{Introduction}

Traumatic brain injury (TBI) is a global health issue that negatively impacts individuals with brain injury, their caregivers, and society. ${ }^{1}$ Therefore, attending to the needs of critically ill patients is an essential and necessary step in providing appropriate care for both the patient and the caregivers. ${ }^{2}$ Lack of proper knowledge and nursing techniques cause relatives at home to provide improper care to the TBI survivors, hence, further complications may arise in the form of secondary bacterial infections. ${ }^{3}$ The primary responsibility of care is placed on the shoulders of the caregiver. Due to the challenges TBI survivors face, caring for them at home might be difficult, especially during the pandemic. Therefore, it is essential that caregivers are provided with essential knowledge on how to take care if their loved ones. ${ }^{4}$ Several studies state that globally, TBI survivors have a lower life expectancy than the general population with significant variations in the physical, cognitive, and social aspects of recovery, which make the prognosis for the individual uncertain. Due to this uncertainty, caregivers often have urgent need for information, emotional support, and care involvement, which could improve the lives of their patients. ${ }^{5-8}$ The results of brain injury often cause the roles and responsibilities within a family to change, because individuals with TBI often rely on caregivers for long-term and life-long assistance. ${ }^{9}$ Furthermore, during pandemics, opportunists may also arise. Reports during COVID-19 show an increase in unsavory individuals attempting to take advantage of vulnerable people, especially those with traumatic brain injury and other physical impairments. Caregivers should maintain daily contact with the health care providers looking after the survivors and also ask questions frequently. People suffering from brain injury may showcase behavioral changes which caused by the element, this might cause their caregivers' discomfort. Therefore, this study aims to explore the needs of the caregivers of TBI survivors during the COVID-19 pandemic. 


\section{Design and Methods}

The collection of data commenced following the approval of the Kulliyyah Nursing Committee (KON), the University Ethics Committee (IREC), and the approval of Medical Ethics by the Ministry of Health of Malaysia through the NMRR. Consent was obtained from the caregivers, after which they signed consent forms as an agreement to participate in this study. All caregivers were kept anonymous, and all researchers and co-researchers directly involved in the study ensured the confidentiality of the information collected. Furthermore, the information obtained in this study was strictly used for academic purposes.

The participants in this study were caregivers of severe traumatic brain injury survivors and they can be defined as the parent, child, sibling, or spouse of the patient. The targeted caregivers were identified from the patient admission records in the Intensive Care Unit. Furthermore, those that fulfilled the inclusion and exclusion criteria were selected through purposive sampling. Caregivers looking after patients with severe TBI were assigned a Glasgow Coma Score (GCS) ranging from 3-9 after the selection. The researcher made an appointment with the participants via phone call and those that agreed to a meeting were given an information sheet by the researcher.

\section{Results and Discussions}

All 10 caregivers gave their written consent for demographic and basic medical information to be collected from either them or the hospital (Table 1). The caregivers participating in this study had varying relationships. However, the most common one found in the group was the father-child relationship.

Overall, three significant themes emerged in the analysis, which will be briefly outlined and described later in greater detail. The thematic analysis identified the following areas: i) support needs; ii) information needs; and iii) developing self-resilience. Furthermore, when respondents' statements were being classified into different categories, it was observed that some categories were interrelated. During this study, participants were asked questions which facilitated the exploration of the common needs of care during the recovery process of the patients.

\section{Theme 1. Support needed}

Family involvement in caring for the TBI survivors. Three participants discussed the importance of family involvement especially during the pandemic (COVID-19). Support from family and relatives was often featured in the interview data. Examples are shown in the following quotations:

"My brother and I always take turns looking after my father, but because of the COVID-19, my brother can't return home because he needs to obey the Control Movement Order by government" (participant no. 1)

"I really need my family members to return back home, taking care of my younger sister alone makes me tired, and because I can't rest, I worry that they can't enter the state due to the pandemic" (participant no.3)

"During this period (COVID-19), I had to take a leave without pay from my job and after a few days, my boss called me and asked me to resign. Although the reality is difficult to accept, I need to look after my brother daily" (participant no. 7)

Need for spiritual assistance within the care-giving roles was discussed and prayer was the most mentioned topic.

"I asked for help when reciting a prayer to our son each time we talk through a video call or voice call, because COVID-19 makes it difficult to see him" (Participant no. 3)

"I hope that something good will happen to him. We always hope that he will get better by reciting a prayer at home" (Participant no. 9)

\section{Psychological support needs focus on positive thinking and dis- traction}

The caregivers are always willing to take care of patients and show their strength when caring for them especially during the pandemic situation, even though their emotions might be unstable.

"I was under stress, but I still want to give him the best" (participant no. 8)

"She always thinks her son's health is getting worse poor, and she cries all the time. Therefore, I advised her to 'isthifar' and remember Allah" (participant no. 5)

"I'm afraid to look at his condition, but I need to show that I'm

Table 1. Caregivers' socio-demographic information.

\begin{tabular}{lcccc} 
No. & Relationship & Family caregiver age & Occupation/sector & Highest education level \\
1. & Father & 53 & Technician & College \\
2. & Father & 47 & Government & University \\
\hline 3. & Sister & 36 & Private & University \\
4. & Brother & 34 & Student & University \\
\hline 5. & Father & 56 & self-worker & LCE \\
6. & Daughter & 28 & Salesgirl & University \\
\hline 7. & Daughter & 45 & Government & University \\
8. & Mother & 47 & Government & College \\
\hline 9. & Mother & 58 & Housewife & LCE \\
10. & Father & 54 & Labor & LCE \\
\hline
\end{tabular}

LCE, Low certificate examination. 
strong when taking care of him, that is why I try my best to avoid crying in front of him" (participant no. 2)

\section{Theme 2. Information needs about care}

The caregiver families always seek information on the exact problems of the patient, because they feel uncertain about how to care for them at home. Therefore, they discussed seeing the healthcare provider when necessary.

"I was thinking, how long will my son be breathing through that tube (Tracheostomy tube)?" (Participant no. 2)

"I'm afraid I'll make the situation worse because I do not have the experience caring a for a person with a head wound and a tube in the nose" (Participant no. 6)

They also discussed how to look after the survivors at home and how to access to someone or a company that provides medical equipment.

"When the doctor told me I could take my sister back home, I asked him (doctor) where can I get information on how to take care of her? Taking care of her is not easy because this is the first time I have done something like this, and there are no medical devices at home" (Participant no. 3)

"I was looking for a company that sold hospital equipment and asked my colleagues if they knew any place that i could refer to" (participant no. 4)

Gaining more knowledge and understanding of the problems of primary caregivers will lead to the provision of more specific and direct support.

\section{Theme 3. Developing self-resilience}

Improving coping skills, help caregivers understand the value of their circumstances and how to better respond to them. The situation helps caregivers develop a sense of resilience and they become more responsive and understanding to their environment. Almost all of the respondents used religious coping techniques as a means dealing with the situation.

"I almost gave up, but when you remember you can still breathe, then your hope can never die, and also remember Allah SWT is always alive to help the living!" (participant no .6)

"I recited additional prayers at night, such as the midnight, 'tahajjud', and 'hajat' prayers." (participant no .2)

\section{Emotional feelings}

Caregivers usually express negative feelings such as anxiety towards their current situation and losing hope after facing many challenges on caring for the TBI survivor at home. The following quotations are from three participants:

"I sometimes worry about how I was going to cope when carrying my son to the hospital had he been left disabled during the COVID-19 pandemic." (participant no.10)

"Although she supported everyone else and did not show her feelings, she had sleepless nights worrying about her dad's condition" (participant no .7)

Social concerns are one of the important functions of social relationships. The caregivers discussed their social needs, when they are taking care of things at home and plan future endeavors. The values of the neighborhood were highlighted as social concerns by the caregivers. The following quotations show how the neighbors understood and sympathized with them:

"I am lucky to have neighbors who come to visit and help, we are close which means we always meet each other" (participant no. 4)

"Sometimes I worry about what my relatives or friends think of my son, which causes feelings of guilt and shame within me" (participant no. 9)

In this study, the interviews' result focused on the caregiver's needs during the pandemic (COVID-19). The shared experiences from the caregivers on how they cared for the patients at home have provided the researchers with useful information regarding the nursing process when caring for TBI survivors. Consequently, the most crucial part which is the importance of knowing what kind of support is needed was discussed and concluded.

\section{Support needed}

The results suggest that caregivers have similar perspectives and that they need strong support. Their needs are higher when preparing to bring the TBI survivors back home and during their first few months at home. This result is supported by a previous study which stated that sustaining a Traumatic Brain Injury caused the caregiver strain due to the impact such an injury causes to the family and community. ${ }^{5}$ Furthermore, the well-being of the caregiver and family members affect their ability and willingness to care for the injured person. ${ }^{10}$ The social work practitioner needs support which includes thoroughly assessing the survivor's family function with particular attention on gender, family role definitions, the family's ability to appropriately and emotionally respond to each other, and their ability to solve family problems proactively. ${ }^{11}$ Therefore, in addition to providing patient care, caregivers must also play their role in family support by providing information and allowing members to be near the patients. ${ }^{12}$ Another essential need of caregivers taking long-term care of TBI survivors was emotional support. Caregivers and families play an important, all-encompassing role in the care of individuals with TBI. This role is often accompanied by an overwhelming sense of stress experienced by the patient and their entire family. ${ }^{13}$

In several studies, it was reported that caregivers are struggled to manage the behavior of TBI survivors which may become a primary determinant of the caregiver's burden. ${ }^{14-16}$ Furthermore, inadequate preparation of caregivers and patients regarding this personality and behavioral changes can pose a problem. Caregivers may lack the skills to manage, cope, and provide care in TBI rehabilitation. ${ }^{17}$ The results of this study, show that caregivers face difficulty communicating with survivors, and this miscommunication always occurs during the recovery period. This situation caused the caregivers to feel unsatisfied with the respect has being given. The published literature about social concern is always focused on community and environmental support. ${ }^{18}$

The role of friends, relatives, and neighbors is essential in building social support among caretakers as they need significant support regarding social concern. This point is crucial for a deeper integration of social support within the community. Due to this uncertainty, caregivers often need additional information, emotional support, and involvement in care, as they are struggled to adapt to the changes in their life. ${ }^{19}$ The close relatives felt they were lonelier than they were before the accident, mainly because now they only focus on the person with TBI. Supporting close relatives in their new situation, will help them empower the person with TBI to manage their altered daily life. ${ }^{19}$ For example, a study found that perceived support was more beneficial to caregivers, than enacted support, where enacted support referred to the actual helping act 
and perceived social support referred to an individual's cognitive appraisal of his/her social connections. ${ }^{20}$ In this research, it was found that social concerns played a critical role in the adaptation of caregivers to their situation of caring for TBI patients during the pandemic. Therefore, without the social support, caregivers feel that they are not a part of the community.

\section{Information needs about care}

Lack of information provided, is one of the most frequently cited problems within studies that reflect the experiences of caregivers. ${ }^{21}$ Caregivers usually have no standard information on how to take care of patients with TBI. Based on this finding, the caregiver needs to be provided with information regarding the care of TBI patients at home. This will further encourage healthcare professionals to help caregivers prepare themselves for the responsibility of caring for their loved ones. Therefore, caregivers should also play their roles by upgrading their care knowledge by seeking information and allowing patients to be near them during the acute phase of the ailment. ${ }^{22}$

\section{Adaptation to a new care environment}

In addition to the stress associated with their circumstances, caregivers face the additional pressure of being sole providers of their loved ones during the pandemic. During this time, the caregiver only leaves the house for essential purposes. Subsequently, family members from other places com to visit and meet with the caregivers to ensure that the TBI survivors as well. They may also deliver food and perform any required jobs in a short time. People that have family members with traumatic brain injury may struggle to explain why the pandemic causes certain modifications in the home. But the shift in roles and relationships of both parties can cause the caregiver to develop a resilience mechanism. ${ }^{23-25}$

\section{Conclusions}

This study's findings reveal the importance of exploring the general needs of severe Traumatic Brain Injury survivors. Caregivers always need full support while at home, and the family members also need to assist and support these caregivers in solving daily problems caused when dealing with TBI survivors. Moreover, during the rehabilitation phase, caregivers are responsible for fulfilling the additional roles of unconscious survivors. Caregivers of TBI survivors have unique needs because the ailment is always a sudden event. Therefore, this study's results can be used as a guide to the healthcare provider in order to better support the caregivers of TBI survivors. In this study, caregivers shared several of their unfulfilled needs concerning support. Furthermore, additional strain due to the pandemic, cause caregivers to face extra challenges. Caregivers living with TBI survivors are finding new ways of adapting to the present context which causes the changes in the social and health care conditions. Future studies are needed to reveal the other consequences of traumatic brain injury, and after such results are revealed, several needs can be met through support programs.
Correspondence: Hamidah Othman, Critical Care Nursing Department, Kulliyyah of Nursing, International Islamic University Malaysia (IIUM), 25200, Kuantan, Pahang, Malaysia.

Tel. +60.9570 .7300 Ext. $7201 /+60.132036156$.

E-mail: hamidahothman78@gmail.com

Acknowledgments: The authors are grateful to the research participants for their time and sincerity. The authors are also grateful to the Supervisory committee for the helpful discussions and assistance provided in identifying the themes in the data analysis.

Key words: Caregivers; traumatic brain injury; pandemic COVID-19; resilience; coping strategy.

Contributions: The authors are responsible for the content and writing of the paper.

Conflict of interests: The authors declare no potential conflict of interest.

Funding: Publication of this research was funded by the Fundamental Research Grant Scheme (FRGS) from the Ministry of Education (MOE) Malaysia: project ID: 15/221/0462.

Ethical approval: The study was approved by the Kulliyyah Nursing Committee (KON), the University Ethics Committee (IREC), and of Medical Ethics by the Ministry of Health of Malaysia through the NMRR.

Conference presentation: Part of this study was presented at the $1^{\text {st }}$ International Nursing and Health Sciences Symposium, November $13^{\text {th }}$ to $15^{\text {th }} 2020$, Brawijaya University, Malang, Indonesia.

Received for publication: 20 January 2021

Accepted for publication: 22 March 2021.

oCopyright: the Author(s), 2021

Licensee PAGEPress, Italy

Journal of Public Health Research 2021;10:2205

doi:10.4081/jphr.2021.2205

This work is licensed under a Creative Commons Attribution NonCommercial 4.0 License (CC BY-NC 4.0).

\section{References}

1. Savicki LE. Collaborative referencing in traumatic brain injury. Thesis Degree, University of Iowa; 2012.

2. Kynoch K, Chang A, Coyer F. Evidence to practice gap : difficulties in implementing evidence-based recommendations to meet family needs in an adult intensive care unit. JBI Evid Implemen 2012;10:272.

3. Minnes P, Woodford L, Carlson P, et al. The needs of aging parents caring for an adult with acquired brain injury. Can J Aging 2010;29:185-92.

4. Rodakowski J, Skidmore ER, Rogers JC, et al. Role of social support in predicting caregiver burden. Archi Phys Med Rehabil 2012;93:229-36.

5. Liu W, Zhu J, Liu J, et al. Psychological state and needs of family member caregivers for victims of traumatic brain injury: A cross-sectional descriptive study. Int J Nursing Sci 2015;2:231-6.

6. Norup A, Perrin PB, Cuberos-Urbano G, et al. Family needs after brain injury: A cross cultural study. Neuro Rehabil 2015;36:203-14.

7. O'Callaghan AM, McAllister L, Wilson L. Experiences of 
care: Perspectives of carers of adults with traumatic brain injury. Int J Speech Lang Pathol 2011;13:218-26.

8. Mbakile-Mahlanza L, Manderson L, Downing M, et al. Family caregiving of individuals with traumatic brain injury in Botswana. Disabil Rehabil 2017;39:559-67.

9. Dillahunt-aspillaga C, Jorgensen-smith $\mathrm{T}$, Ehlke S, et al. Traumatic brain injury: Unmet support needs of caregivers and families in Florida. PloS One 2013;8:1-10.

10. Vangel SJJ, Rapport LJ, Hanks RA. Effects of family and caregiver psychosocial functioning on outcomes in persons with traumatic brain injury. J Head Trauma Rehabil 2011;26:20-9.

11. Barclay DA. Family functioning, psychosocial stress, and goal attainment in brain injury rehabilitation. J Soc Work Disabil Rehabil 2013;12:159-75.

12. Soury-Lavergne A, Hauchard I, Dray S, et al. Survey of caregiver opinions on the practicalities of family-centred care in intensive care units. J Clin Nurs 2012;21:1060-7.

13. Arango-Lasprilla JC, Quijano MC, Aponte M, et al. Family needs in caregivers of individuals with traumatic brain injury from Colombia, South America. Brain Inj 2010;24:1017-26.

14. Chronister J, Chan F, Sasson-Gelman EJ, et al. The association of stress-coping variables to quality of life among caregivers of individuals with traumatic brain injury. Neuro Rehabil 2010;27:49-62.

15. Norup A, Welling KL, Qvist J, et al. Depression, anxiety and quality-of-life among relatives of patients with severe brain injury: the acute phase. Brain Injury 2012;26:1192-200.

16. Bayen E, Pradat-Diehl P, Jourdan C, et al. Predictors of informal care burden 1 year after a severe traumatic brain injury: results from the Paris-TBI study. J Head Trauma Rehabil 2013;28:408-418.
17. Liu W, Zhu J, Liu J, et al. Psychological state and needs of family member caregivers for victims of traumatic brain injury: A cross-sectional descriptive study. Int J Nurs Sci 2015;2:231-6.

18. So S. Transition as experienced by close relatives of people with traumatic brain injury. J Neurosci Nurs 2011;43:253-60.

19. Webster J, Taylor A, Balchin R. Traumatic brain injury, the hidden pandemic: A focused response to family and patient experiences and needs. South Afr Med J 2015;105:195-8.

20. Boycott N, Yeoman P, Vesey P. Factors associated with strain in carers of people with traumatic brain injury. J Head Trauma Rehabil 2013;28:106-15.

21. Chronister J, Chan F, Sasson-Gelman E, et al. The association of stress-coping variables to quality of life among caregivers of individuals with traumatic brain injury. Neuro Rehabil 2010;27:49-62.

22. Hassan S, Khaw W, Rosna A, et al. Traumatic brain injury: caregivers' problems and needs. JNMA J Nepal Med Assoc 2011;50:53-5.

23. Anderson MI, Daher M, Simpson GK. A predictive model of resilience among family caregivers supporting relatives with traumatic brain injury (TBI): A structural equation modelling approach. Neuropsychol Rehabil 2020;30:1925-46.

24. Simpson G, Jones K. How important is resilience among family members supporting relatives with traumatic brain injury or spinal cord injury? Clin Rehabil 2012;27:367-77.

25. Las Hayas C, López de Arroyabe E, Calvete E. Resilience in family caregivers of persons with acquired brain injury. Rehabil Psychol 2015;60:295-302. 